\title{
THE DYNAMICS OF LEUKOPOIESIS AND LEUKOCYTOSIS, AS STUDIED BY LEUKOPHERESIS AND ISOTOPIC TECHNIQUES ${ }^{1}$
}

\author{
By CHARLES G. CRADDOCK, JR.,2 SEYMOUR PERRY, AND JOHN S. LAWRENCE \\ WITH THE TECHNICAL ASSISTANCE OF MARY H. BAKER AND GLORIA PAUL \\ (From the Department of Medicine, School of Medicine, University of California, and the \\ Hematology Research Laboratory, Veterans Administration Center, \\ Los Angeles, Calif.)
}

(Submitted for publication July 25, 1955; accepted November 7, 1955)

Previous studies $(1,2)$ in normal and irradiated dogs employing the technique of leukopheresis have suggested some hitherto unknown relationships between the number of circulating leukocytes and the reserves of these cells elsewhere in the body. By way of introduction and as background for the data to be presented it is necessary to review briefly and interpret some of these findings.

The acute response to the production of severe leukopenia by leukopheresis is qualitatively similar from animal to animal and is divisible into three phases. When leukopheresis is terminated there is a delay before the peripheral white blood cell count begins to rise (phase I). Following this there occurs a linear rise in peripheral blood count over a six to eight-hour period (phase II). After a slower rise to a peak peripheral white cell count, usually 150 to 200 per cent of baseline, the white cell count stabilizes at this new level for several hours (usually 24 to 36 ) ; this last period is denoted as phase III. Phase I, or the delay period before the white blood cell count begins to rise, is not observed unless the count is reduced to 1,500 or 1,000 cells per $\mathrm{mm}^{3}$. Less marked degrees of leukopenia, induced by slower rates of clearance of white cells from the peripheral blood, may be maintained by continued leukopheresis for several hours but are not followed by the period of sustained leukopenia (phase I). This is in spite of the removal of many more cells than would be obtained during a short, rapid leukopheresis which produces marked leukopenia. On the other hand, phase $I$ is observed after marked $\left(1,500\right.$ per $\mathrm{mm}^{3}$ or less) leukopenia no matter how long leuko-

1 This work was supported by grants from Parke-Davis and Co., the U. S. Public Health Service, and Gladys F. Bowyer Fund.

2 Markle Foundation Scholar in Medical Science. pheresis is continued after leukopenia is induced. For these reasons phase $I$ is thought to reflect a more rapid rate of dispersal of leukocytes to the periphery which offsets the accelerated entry of cells from the marrow into the circulation. In other words, the evidence suggests that the change from a stationary leukopenia (phase I) to a rapidly rising level (phase II) is due to a sudden alteration in the rate of distribution of leukocytes to peripheral tissues. Once this "priming" has occurred in phase I, the blood white cell count begins to rise rapidly, reflecting the accelerated entry of leukocytes into the circulating blood.

Phase II represents a period of greatly accelerated entry of leukocytes into the peripheral blood, which continues until the new maximum is reached. Rapid leukopheresis with the removal of large numbers of cells may be carried out for a prolonged period without the production of leukopenia if the procedure is instituted during phase II (see Figure 2).

Phase III represents a period of entry of leukocytes into the peripheral blood which has slowed to approximately that at time zero. Leukopheresis instituted during phase III results in the same degree of leukopenia produced by the same rate of leukopheresis as carried out initially. In most animals this type of response occurs repeatedly until about the third or fourth day. By this time acceleration in the growth of myeloid tissue has occurred so that the delay after cessation of leukopheresis is shorter and the leukocytosis develops more rapidly. The maximal white cell count in the peripheral blood after leukopheresis on the third day varies from animal to animal; in most it is similar to the level after previous leukophereses, in some it is higher and in others it is lower. All animals show a slower, progressive rise to a 


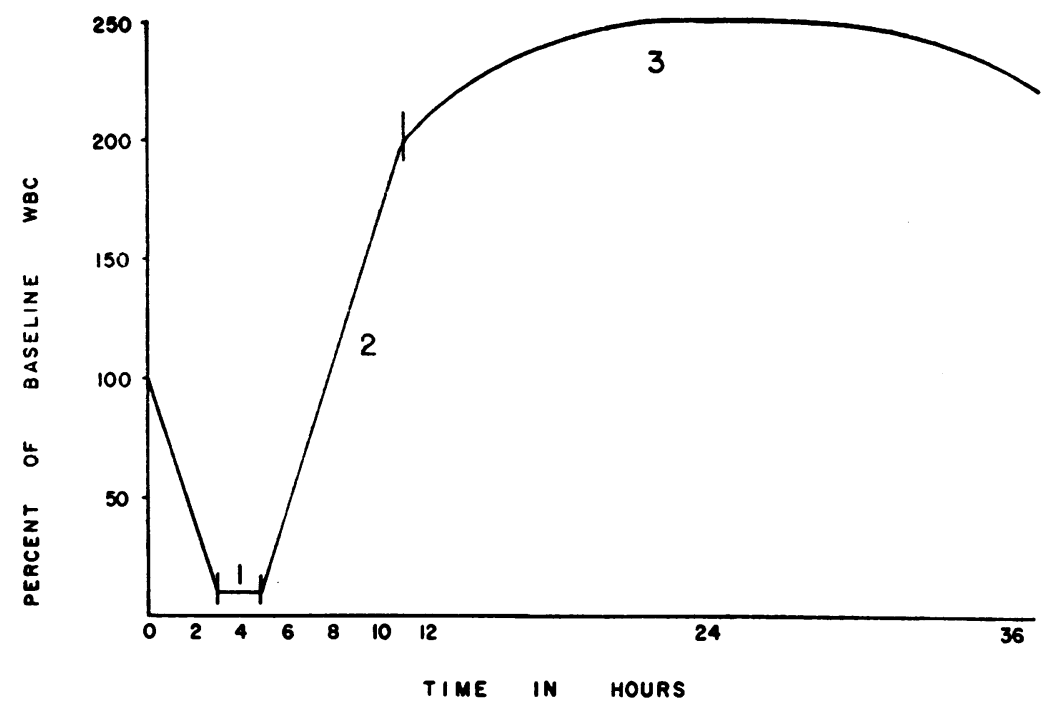

Fig. 1. Schematic Representation of the Typical Response of the Circulating Leukocyte Count to Leukopenia Induced by a Single LEUKOPHERESIS

Removal of leukocytes terminated at beginning of Phase 1 . See text for explanation.

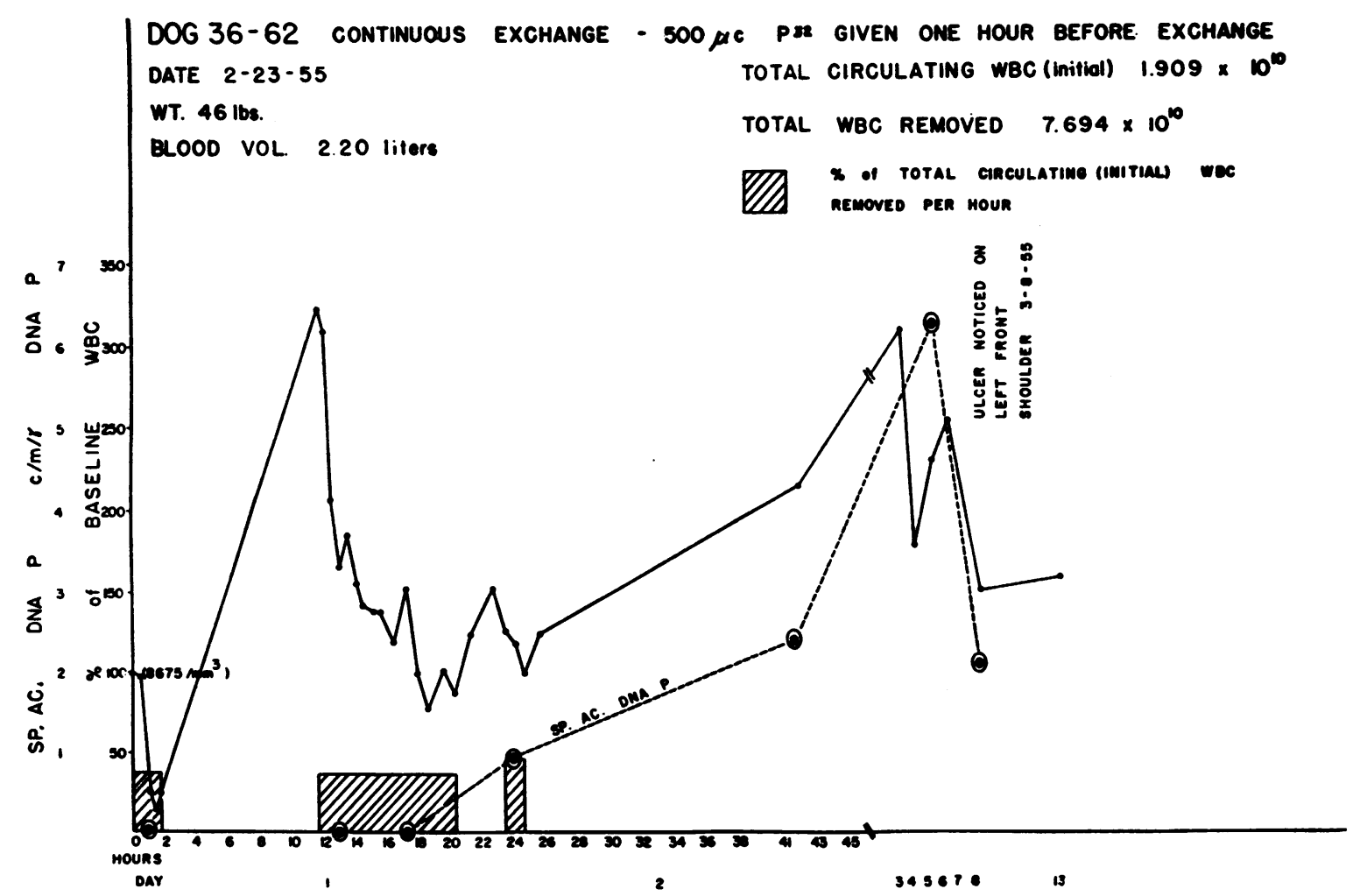

Fig. 2. Induction of Leukopenia by Acute Leukopheresis Followed by Prolonged, Continued LeukoPheresis (Over 8 Hours) Instrtuted during the Period of Developing Leukocytosis Following the Initial DEPLETION

Note that leukopenia was not induced during this time despite removal of over $7 \times 10^{10}$ leukocytes. The data derived from the specific activity of white cell DNA phosphorus (expressed as counts per minute per microgram of DNA phosphorus) indicate that the removed cells were largely unlabeled. 


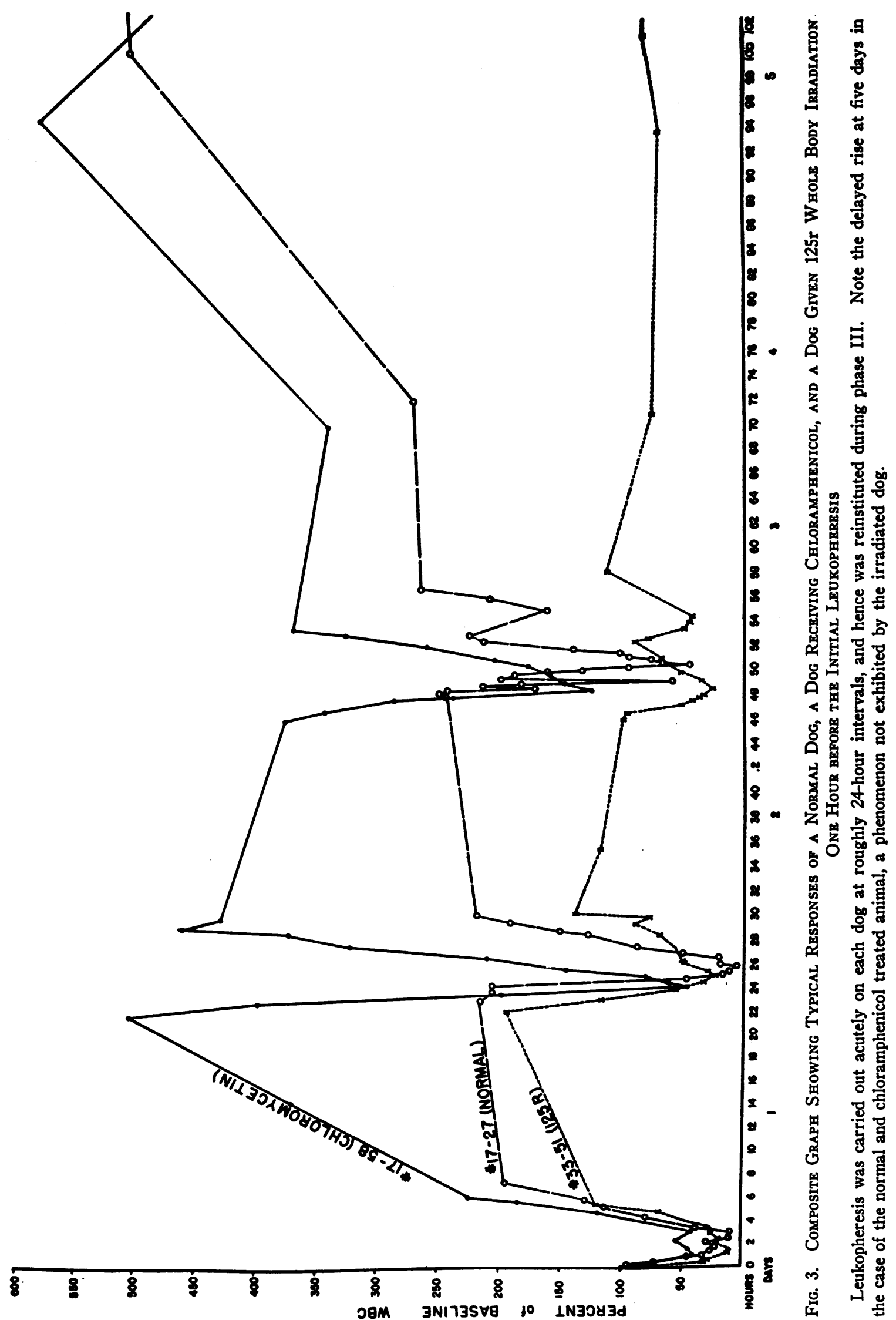


new maximum over the next two to three days (Figure 3). The slope of this delayed rise is similar in normal animals regardless of the level attained during phase II.

The morphology of the peripheral blood leukocytes and the marrow have been correlated with these quantitative changes. There is no change indicative of increasing immaturity of the marrow or circulating leukocytes until the third or fourth day. Undoubtedly some stimulation to leukopoiesis has occurred during this three-day period.

These data suggest that the initial acute response to leukopheresis involves the release of preformed leukocytes from areas of storage. This initial response does not depend upon growth processes. Dogs exposed to LD-50 whole body $\mathrm{x}$-ray behave similarly, though the leukocytosis is quantitatively less, until their marrow has become depleted of myeloid cells. Even after the development of radiation leukopenia, the response to leukopheresis is qualitatively similar, as shown in Figure 5. A fundamental relationship seems to prevail between the leukocyte reserves and the degree of leukocytosis which develops after leukopheresis. The removal of leukocytes from the peripheral blood calls forth the release of cells from the marrow until a new level has been attained. The height of the new level depends upon the size of the reservoir of leukocytes. In the irradiated animal the level becomes progressively lower as the marrow reserve falls. Nevertheless, the same pattern prevails and leukocytes are released into the circulating blood in some sort of proportion to the number of cells held in reserve.

The reserves of preformed leukocytes in the body assume fundamental importance as the source of supply of cells taking part in acute leukocytosis or pyogenic reactions of various sorts. It is, therefore, important to delineate the size and site of such reserves of leukocytes. There are essen-

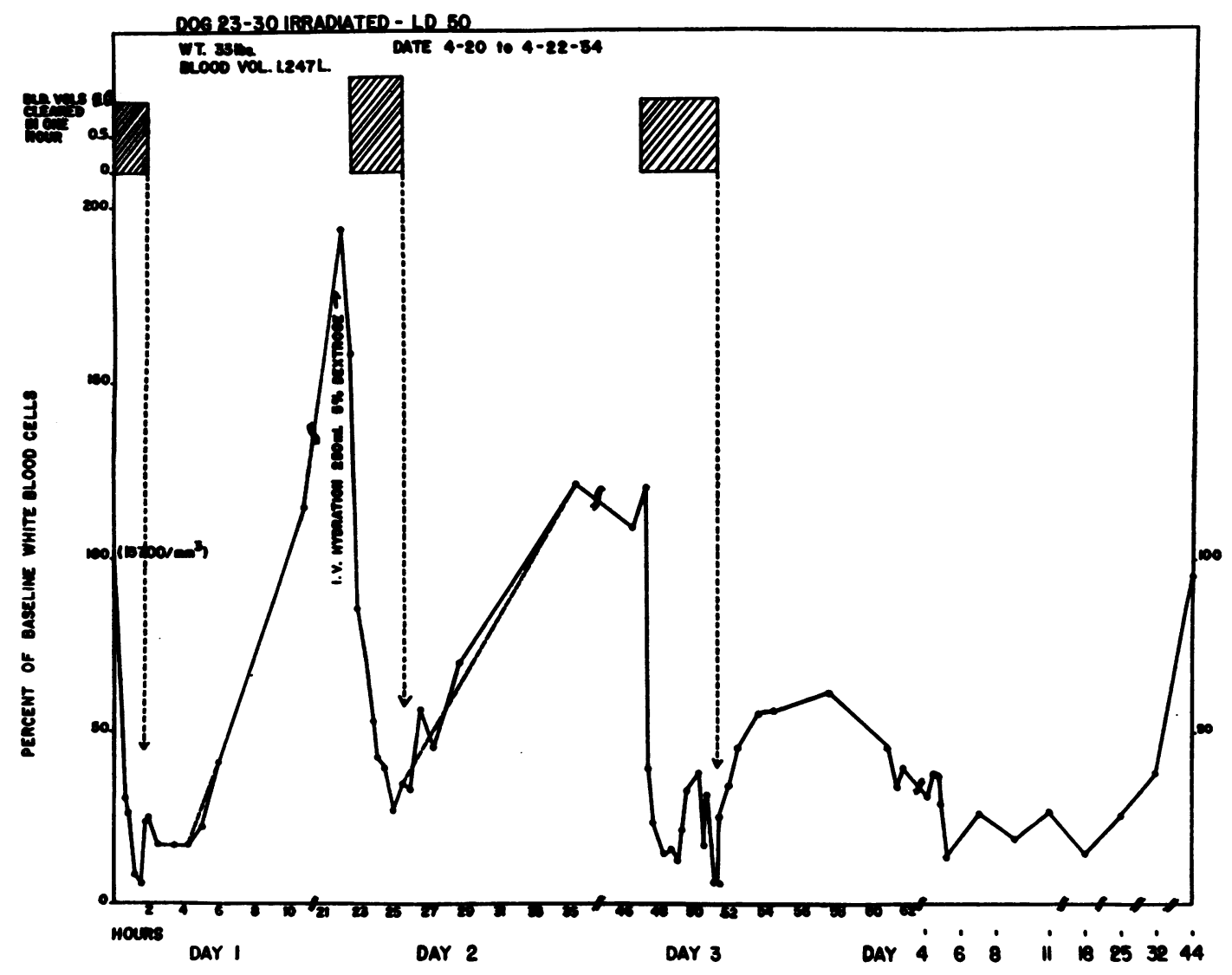

Fig. 4. Response to Leukopheresis Repeated at 24-hour Intervals for Three Days in an Animal Exposed to LD-50 Whole Body Irradiation One Hour Prior to the Initial Leukopheresis 


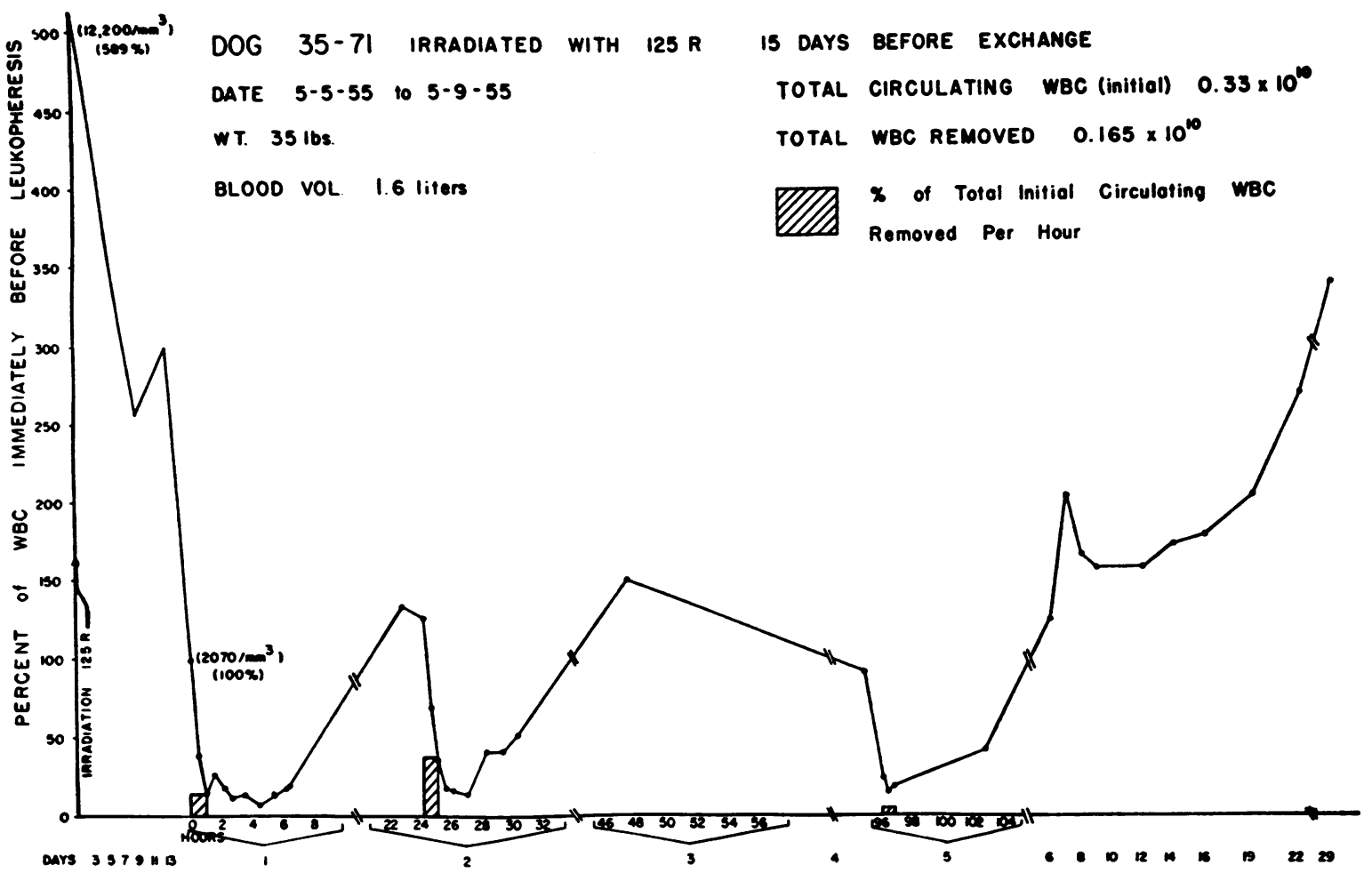

Fig. 5. Leukopheresis of an Anmal Rendered Leukopenic by 125r Whole Body X-Ray Two Weeks Previously

Note that the level of circulating white cells rises after each leukopheresis to a level exceeding baseline, although the rise is much more delayed than in a normal animal.

tially three potential sites of such a "ready reserve": 1) Leukocytes sequestered in various compartments of the vascular bed, 2) leukocytes held within the marrow organ, and 3 ) leukocytes which have migrated out of the vascular bed (peripheral tissues).

It is well known that acute fluctuations in the number of circulating leukocytes occur following hemodynamic alterations in such organs as the lung and spleen. Although the participation of such factors in the response to leukopheresis cannot be entirely eliminated, the uniformity and magnitude of the response in anesthetized animals makes unlikely such events as the leukocytosis of splenic contraction as playing an important role. Epinephrine intravenously did not alter the response (2), and the results in one splenectomized dog (Figure 6) did not differ significantly from normal.

The size of the marrow leukocyte pool has been estimated to be as much as 100 times that of the circulating blood (exclusive of marrow) $(3,4)$.
Certainly our earlier experiments in irradiated dogs $(1,2)$ suggest there are sufficient numbers in the marrow to replace those circulating many times over.

Aside from the marrow the main source of leukocytes to be considered is the extramedullary, extravascular mass of leukocytes. Osgood, for example, (5) estimates that the numbers of leukocytes in such areas are so vast as to be many times the numbers in the marrow and blood. Whether leukocytes which have migrated out of the vascular tree can reenter in sufficient numbers to affect the acute response to leukopheresis is unknown. However, this appears improbable from previous work (2). A heavily irradiated dog, rendered leukopenic by repeated leukopheresis so that fixed agranulocytosis was present showed large collections of leukocytes in peripheral areas of infection in the lung and elsewhere. These leukocytes did not reenter the circulation during or after leukopheresis.

The present experiments were directed pri- 
marily at the question of the source of those leukocytes contributing to the leukocytosis following leukopheresis. For this purpose the rate of entrance of newly formed leukocytes from the marrow has been studied, employing $\mathrm{P}^{32}$ incorporation into leukocyte DNA. Previous works by Kline and Cliffton (6) and Ottesen (7) have indicated the feasibility of this method for determining the mean age, life span and turnover rate of leukocytes. Both groups of investigators noted a delay period of four to six days before labeled myeloid leukocytes appeared in the circulation, attributed to the time required for cells capable of synthesizing DNA at the time of maximum labeling to mature to the point of being released. These workers utilized modifications of the Schmidt-Thannhauser principle of nucleic acid separation. Unfortu- nately, we have been unable to obtain adequate yields of DNA phosphorus uncontaminated by phosphorus from other sources using this method in the study of dog leukocytes. Similar difficulties have been encountered by others ( 8 ). Consequently, a different technique of DNA extraction developed by Dr. Norman Simmons ${ }^{3}$ has been employed. The DNA obtained from leukocytes by this method is highly purified, as determined by spectroscopic, ultracentrifugal, $x$-ray diffraction, and chromatographic techniques.

The leukocyte response to leukopheresis involves myeloid leukocytes almost exclusively (1, 2). Lymphoid cells generally decrease so that the vast majority of leukocytes are of the neutrophilic

${ }^{8}$ Chief, Enzyme Chemistry Section, UCLA Atomic Energy Project.

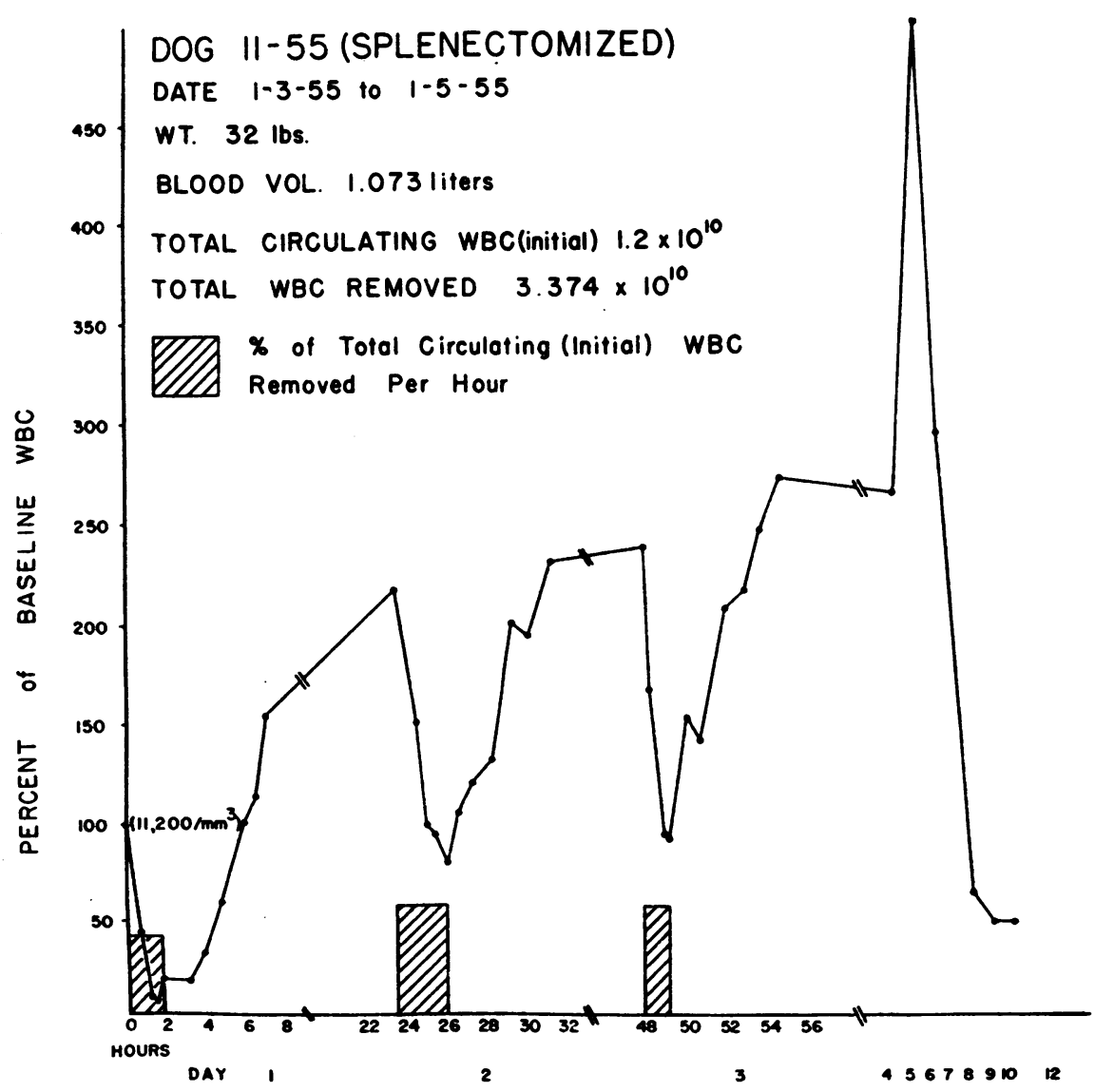

Fig. 6. The Leukocyte Response of a Splenectomized Dog to Repeated LEUKOPHERESIS

The pattern differs in no way from normal. Large numbers of nucleated red cells appeared after the initial leukopheresis; the white cell determinations were corrected for this. 


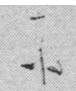

SOLVENT BOUNDARY
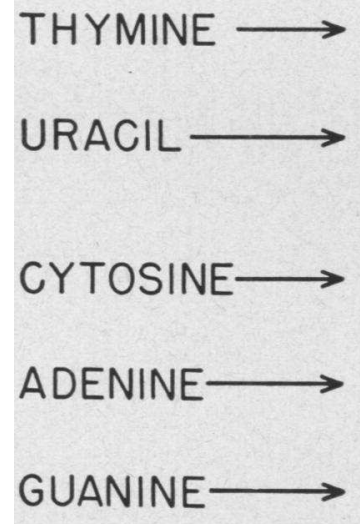

START

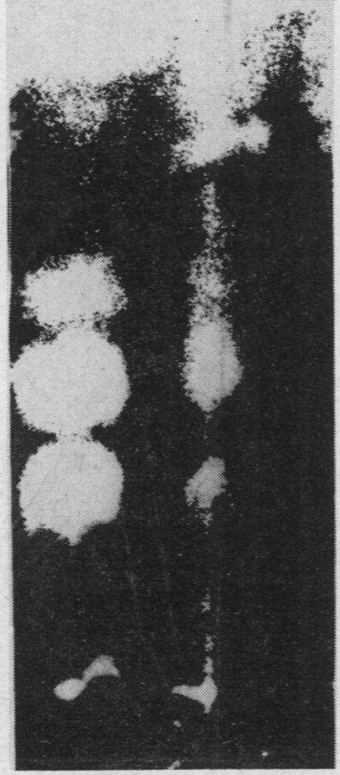

Fig. 7. Paper Chromatogram Showing Purity (LeFt) of Dog Leukocyte DNA Obtained by Method Described in Text as Compared to Virus Preparation Using Schmidt-Thannhauser Technique (Right).

variety. No attempt has been made to obtain more complete separation of lymphocytes and neutrophiles.

\section{METHODS}

Leukocyte suspensions were obtained from dogs. Siliconed glassware was used throughout and the entire procedure was carried out at $4^{\circ} \mathrm{C}$. In animals su. jected to leukopheresis, sufficient numbers of leukocytes were obtained so that separation by simple centrifugation was adequate. In the case of less rich sources, leukocytes were separated from whole blood by means of dextran (Commercial Solvents, av. molecular wt. 120,000). Fifteen $\mathrm{ml}$. of 6 per cent dextran per $25 \mathrm{ml}$. blood was found to give optimal separation. After sedimentation of the red cells the supernatant plasma was centrifuged at 800 to 1,000 r.p.m. for 15 minutes in an International 2 centrifuge to pack the leukocytes and leave the platelets in suspension. After washing twice with saline the leukocytes were diluted to a specific volume, mixed thoroughly and counted in quadruplicate. The theoretical amount of DNA in the cell suspension could thus be calculated.
The leukocytes were then washed with 1 per cent citric acid until the supernate was clear and then homogenized for 5 minutes in 1 per cent citric acid. They were then washed twice, or until the supernatant fluid was clear, with 0.2 per cent citric acid and twice with cold saline. The separated nuclei were subjected to a procedure involving the dissociation of nucleoprotein with 30 per cent sodium xylene sulfonate at $\mathrm{pH} 7$, deproteinization by dilution, filtration, and adjustment of $\mathrm{pH}$ to 3.8 and precipitation of the DNA from neutral solution with isopropyl alcohol. The details of this procedure will be published subsequently by Dr. Simmons and will be the subject of a separate report concerning leukocyte nucleic acids from this laboratory.

The yield of DNA was determined by the diphenylamine reaction and by ultraviolet spectroscopy using pure DNA supplied by Dr. Simmons as standard. Organic and inorganic phosphorus determinations were made on each specimen and in all instances the former checked closely with the yield of DNA. Inorganic phosphorus amounted to less than 10 per cent and the counts of radioactivity were corrected for the small amount present. RNA contamination, as determined by the orcinol reaction, was less than 5 per cent. Quantitative chromatography 4 showed negligible RNA contamination on selected samples. An example of a chromatogram is shown in Figure 7.

The $\mathrm{P}^{32}$ employed was obtained from Abbott Laboratories. $\mathrm{P}^{32}$ counting was carried out in the Radioisotope Section, Research Division, Sawtelle Veterans Administration Hospital using a Tracerlab Superscaler and a TGC-2 Geiger-Muller Tube with a statistical reproducibility of \pm 1 to 2 per cent. All values have been corrected for decay.

$\mathrm{P}^{32}$ was administered as disodium phosphate intravenously in the amount of $500 \mu \mathrm{c}$. regardless of the size of the animal. (The dogs ranged in weight from 30 to 50

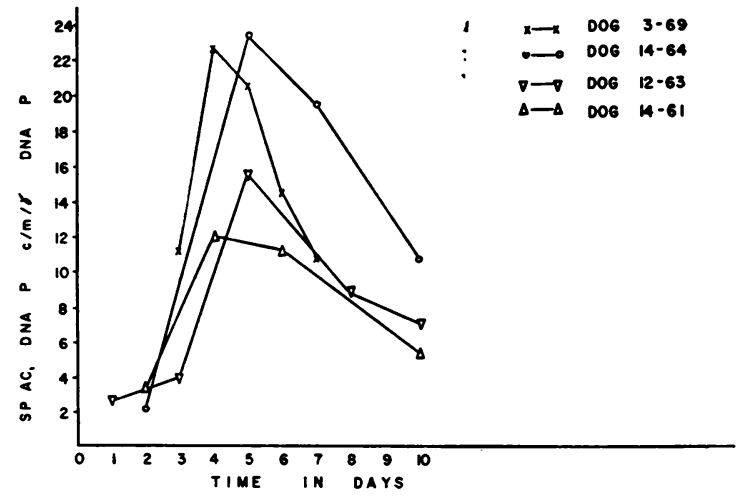

Fig. 8. Specific Activity of DNA Phosphorus in Leukocytes Sampled at Intervals after Administration of 500 Microcuries $P^{83}$ at Time 0 to Four Normal Dogs

These animals were not leukopheresed.

${ }^{4}$ Dr. Gian L. Turco, Research Fellow, California Institute of Technology, graciously performed these studies for us. 


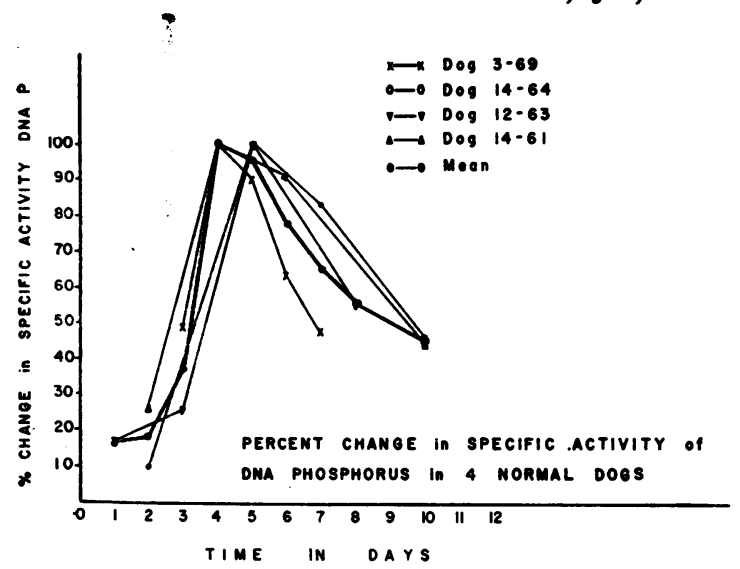

Fig. 9. Same Data Shown in Figure 8 Plotted as Per Cent of the Maximum Specific Activity

lbs.) Those animals who were not subjected to leukopheresis were bled from the jugular vein under sterile conditions, into ACD-dextran solution. After separation of the leukocytes, the red cells were readministered at the time of the next bleeding. All animals withstood the procedure without showing ill effects. No hematologic depression was noticed. Although it is most probable that the amount of irradiation delivered by the $P^{\text {as }}$ has some effect on the processes of cellular growth, the use of the technique in acute studies of differences in entry rate, distribution, and source of leukocytes should be valid.

\section{RESULTS}

Figures 8 and 9 show the results in four normal dogs whose leukocytes were sampled at intervals after administration of the isotope. These dogs were not subjected to leukopheresis. The curves are not comparable from animal to animal in terms

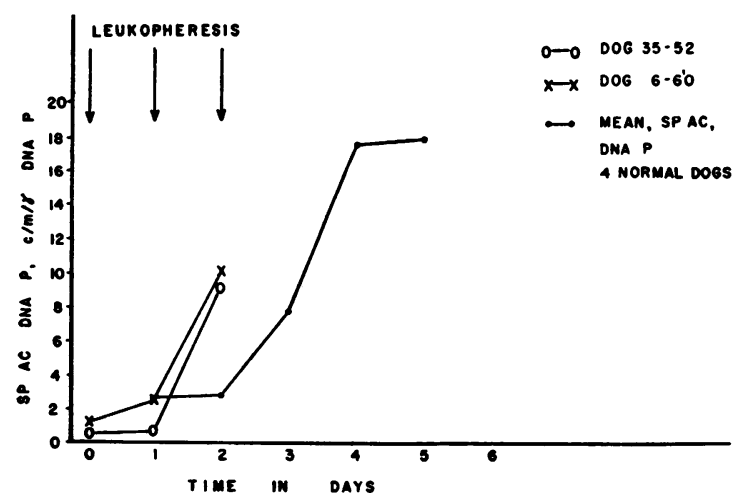

Fig. 10. Acceleration in the Rate of Entrance of Leurocytes Containing DNA Labeled with $\mathbf{P}^{2}, 500$ uc. $P^{*}$ Administered at Time 0

Leukopheresis carried out in $\operatorname{dog} 35-52$ and $6-60$ at indicated points resulting in the typical normal leukocyte response. of the amount of $\mathrm{P}^{32}$ incorporated into DNA since no attempt was made to administer $\mathrm{P}^{32}$ on a weight or blood volume basis. However, the form of the curve for each animal is similar. The mean curve (Figure 9) resembles those observed by others in humans. The rise in specific activity is earlier than in human experiments and the logarithmic curve reaches its maximum on the fourth to fifth day, followed by a brief plateau. Sufficient observations have not been made to describe fully the descending portion of the curve and for the purposes of these experiments emphasis has been placed on the rising portion of the curve as showing the time of appearance of labeled cells in the circulating blood. Calculations of mean cell age

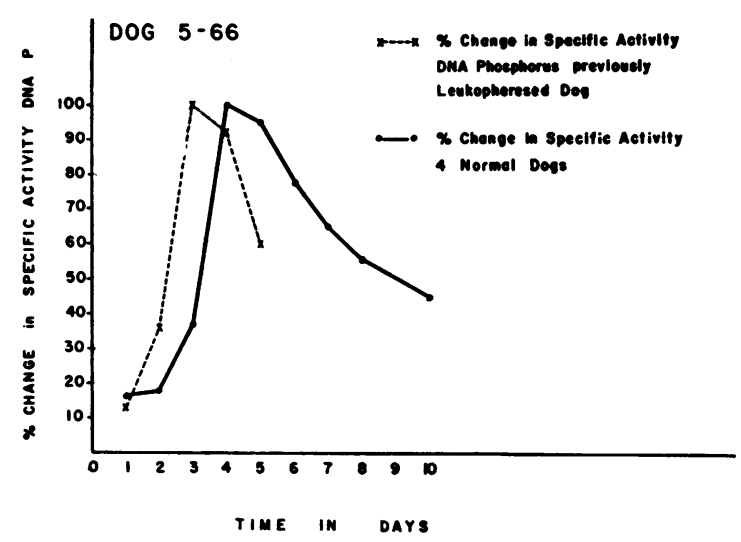

Fig. 11. The Rate of Appearance of Leukocytes with $P^{2 s}$ Labeled DNA into the Peripheral Blood in an Animal Leukopheresed for Three Days Prior to Administration of $500 \mu \mathrm{C}$. P P $^{2}$ Intravenously as ComPared to the Curve for Normal Dogs not Subjected TO LEUKOPHERESIS

and survival time have not been attempted from these limited data, but they suggest a shorter life span for dog leukocytes than that reported for human white cells $(6,7)$.

If leukopheresis is carried out within two hours after administration of $\mathrm{P}^{32}$ the removed cells contain no labeled DNA. Furthermore the cells removed during leukopheresis 24 hours later are still unlabeled, even if large numbers of cells are removed, as is shown in Figure 2. However, repeated leukopheresis does lead to some acceleration in the time of appearance of significantly labeled leukocytes, as is shown in Figure 10. These results are compatible with a large marrow pool of mature leukocytes which precede the entry of those cells which incorporated $\mathrm{P}^{32}$ during the 
process of DNA synthesis. The same type of data is shown in more detail in Figure 2.

Figure 11 shows the curve of specific activity of DNA phosphorus of a normal dog previously subjected to leukopheresis for 3 days before giving the isotope. The maximum of specific activity occurred 24 hours earlier than that of normal dogs not subjected to leukopheresis which suggests the more rapid release of leukocytes from the marrow at this time. This would be expected from the stimulation of leukopoiesis brought about by leukopheresis on three successive days.

Leukopheresis was carried out in three normal animals at a time when those leukocytes whose DNA was maximally labeled with $\mathrm{P}^{32}$ were still in the marrow but shortly before they would be normally released in significant numbers. These dogs were leukopheresed on the third day after being given $500 \mu^{c}$. $\mathrm{P}^{32}$ intravenously. At this point those cells coming from the marrow should have a high specific activity, while cells which were in peripheral areas of storage should con- tain negligible amounts of $\mathrm{P}^{32}$ labeled DNA. Because of this difference in degree of labeling of marrow and peripheral leukocyte pools it should be possible to determine the source of leukocytes contributing to the response to leukopheresis.

Figures 12 and 13 show the results in two of these animals. The third showed an identical response to Figure 13. All dogs showed a relatively low specific activity of leukocyte DNA P in those cells removed during the first leukopheresis. There was a marked rise in specific activity of circulating leukocyte DNA $P$ during the next few hours when the leukocyte count rose after cessation of leukopheresis. It is clear, therefore, that a high percentage of the cells contributing to phase II came from the labeled marrow leukocyte pool. Both dogs showed a fall in specific activity of leukocyte DNA $P$ from this peak the next day. Dog 20-72 (Figure 12) showed very little change in specific activity incident to the second or third leukopheresis. However, Dog 36-70 (Figure 13), after the second leukopheresis, showed a repetition

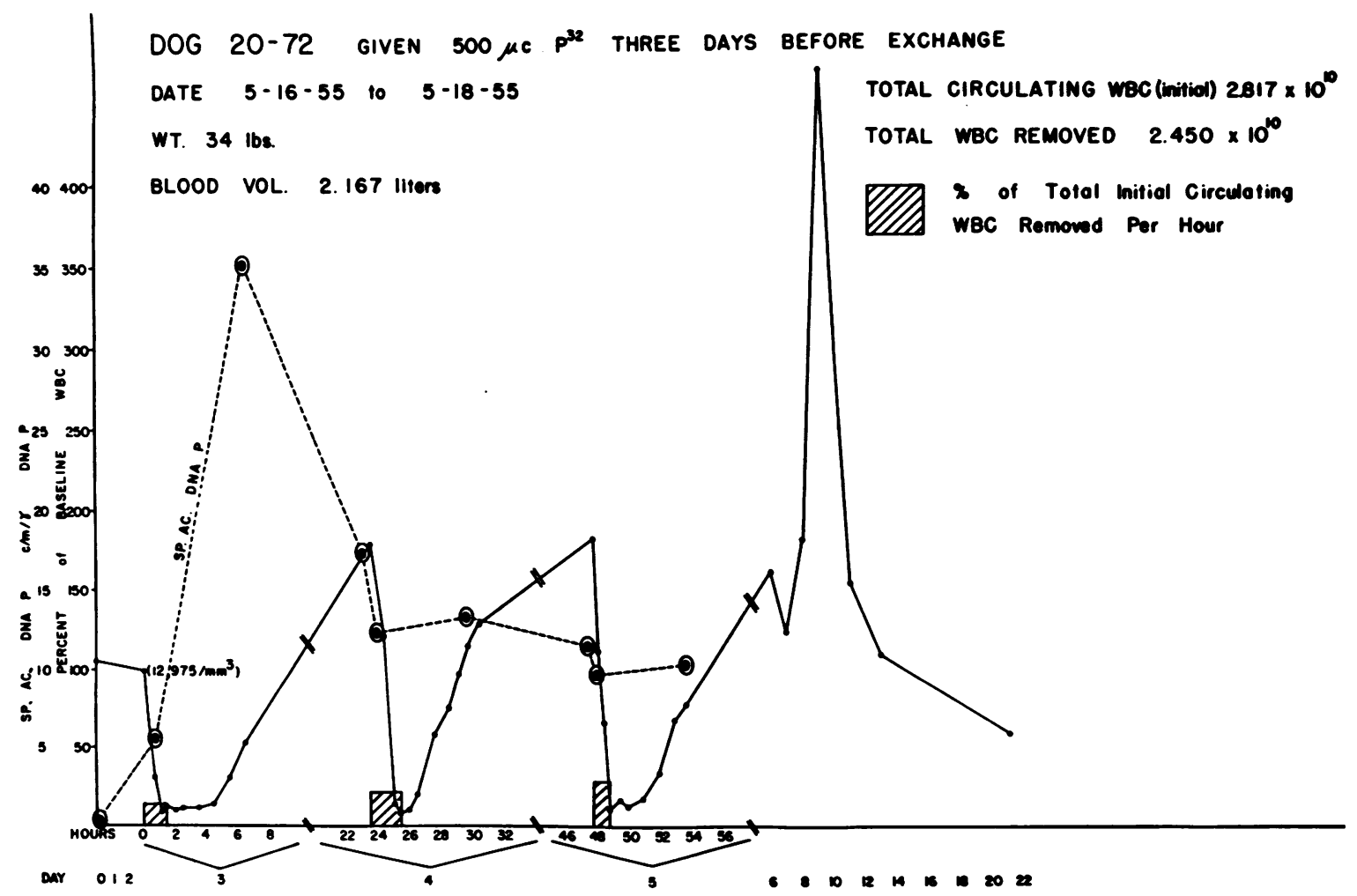

Fig. 12. The Response of a Normal Animal to Repeated Leukopheresis Instituted Three Days after ADMinistration OF $500 \mu \mathrm{c}$. $\mathrm{P}^{32}$ INTRAVENOUSLY

Note the marked rise in specific activity of DNA $P$ in those cells contributing to the leukocytosis after the first leukopheresis. 


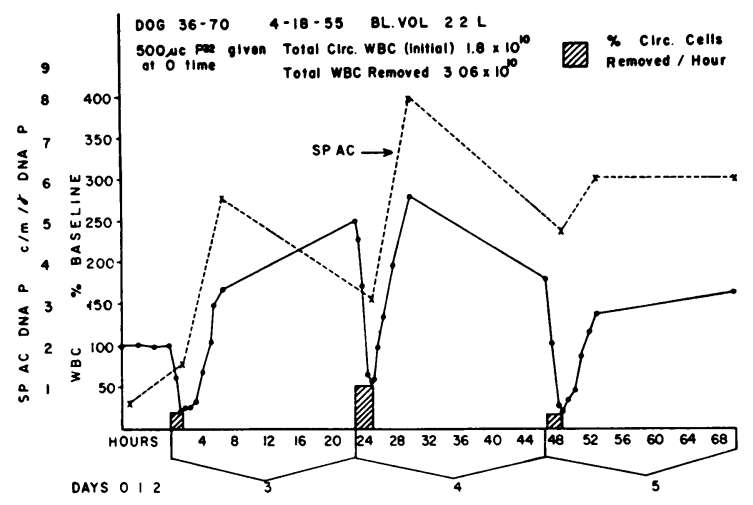

Fig. 13. The Response of a Normal Animal to Repeated Leukopheresis Instituted Three Days after Administration of $500 \mu_{\mathrm{c}}$. $\mathrm{P}^{32}$

Note the rise in specific activity of DNA P during the period of developing leukocytosis after the first two leukophereses.

of the rise in specific activity observed after the first leukopheresis. Whether or not most of the maximally labeled cells appear after the first leukopheresis or later, as in the case of this dog probably depends upon the timing of the procedure.

Both animals exhibited a fall in specific activity of leukocyte DNA P during leukopheresis. This suggests that leukocytes which are older than the average circulating cell population contribute to the mass of cells removed during leukopheresis. The marked rise in specific activity of DNA $\mathrm{P}$ after leukopheresis on one occasion in Dog 20-72 and on two occasions in Dog 36-70 indicate that cells were contributed from the highly labeled marrow pool in much higher percentage than from the relatively unlabeled peripheral tissues.

\section{DISCUSSION}

These data indicate the feasibility of experiments on leukocytes employing a method of DNA extraction which we believe to be superior to the Schmidt-Thannhauser principle used by most other workers.

Preliminary results indicate a life span for dog leukocytes considerably shorter than those reported for humans.

The changes in the concentration in the peripheral blood of white cells having DNA labeled with $\mathrm{P}^{32}$ support the concept, presented earlier in this paper, that the bulk of cells contributing to the leukocytosis after leukopheresis are released from the marrow and do not come from extravascular and extramedullary areas. Therefore, the re- sponse to leukopheresis is believed to represent an accurate measure of the capacity of the marrow to supply the peripheral blood with leukocytes. These findings may be correlated with previous data in irradiated animals (2) to indicate that the marrow represents the main, if not the only, reserve supply of leukocytes available for release into the circulation during leukocytosis of the type observed here.

This does not necessarily mean that leukocytosis can occur only by means of accelerated release of cells from the marrow. There is ample evidence indicating the importance of redistribution of leukocytes within the vascular compartment secondary to various hemodynamic alterations, causing fluctuations in peripheral blood white cell concentration (e.g., exercise, epinephrine). Other types of leukocytosis have not been studied by techniques similar to those employed here. It may be that the leukocytosis subsequent to leukopheresis is unique in its mechanisms. Nevertheless, it has revealed an interesting and intricate relationship between the utilization rate of peripheral blood leukocytes and the release of cells from the marrow. This concept is presented in diagrammatic form in Figure 14.

The rate of development and degree of leukocytosis after leukopheresis depend upon the numerical size of the leukocyte reserve. The evidence presented supports the belief that this reserve is primarily within the marrow. Normally the marrow reserve is replaced at such a rate that repeated or continuous leukopheresis as presently applied fails to greatly affect its size. However, damage by irradiation which reduces the replacement of the reserve by growth depresses the response to repeated leukopheresis. The level attained in the blood after leukopheresis becomes progressively less as the size of marrow reserve becomes reduced.

This relationship between the blood level of leukocytes attained after leukopheresis and the size of the marrow leukocyte reserve suggests that the adequacy of the marrow reserve and replacement by growth may be indirectly assessed by this means. Whether other types of stimulation to leukocytosis such as inflammation or infection are associated with a similar relationship between blood and marrow leukocytes remains to be investigated. The data suggest that the release of cells from the marrow is geared to the utilization rate of leuko- 


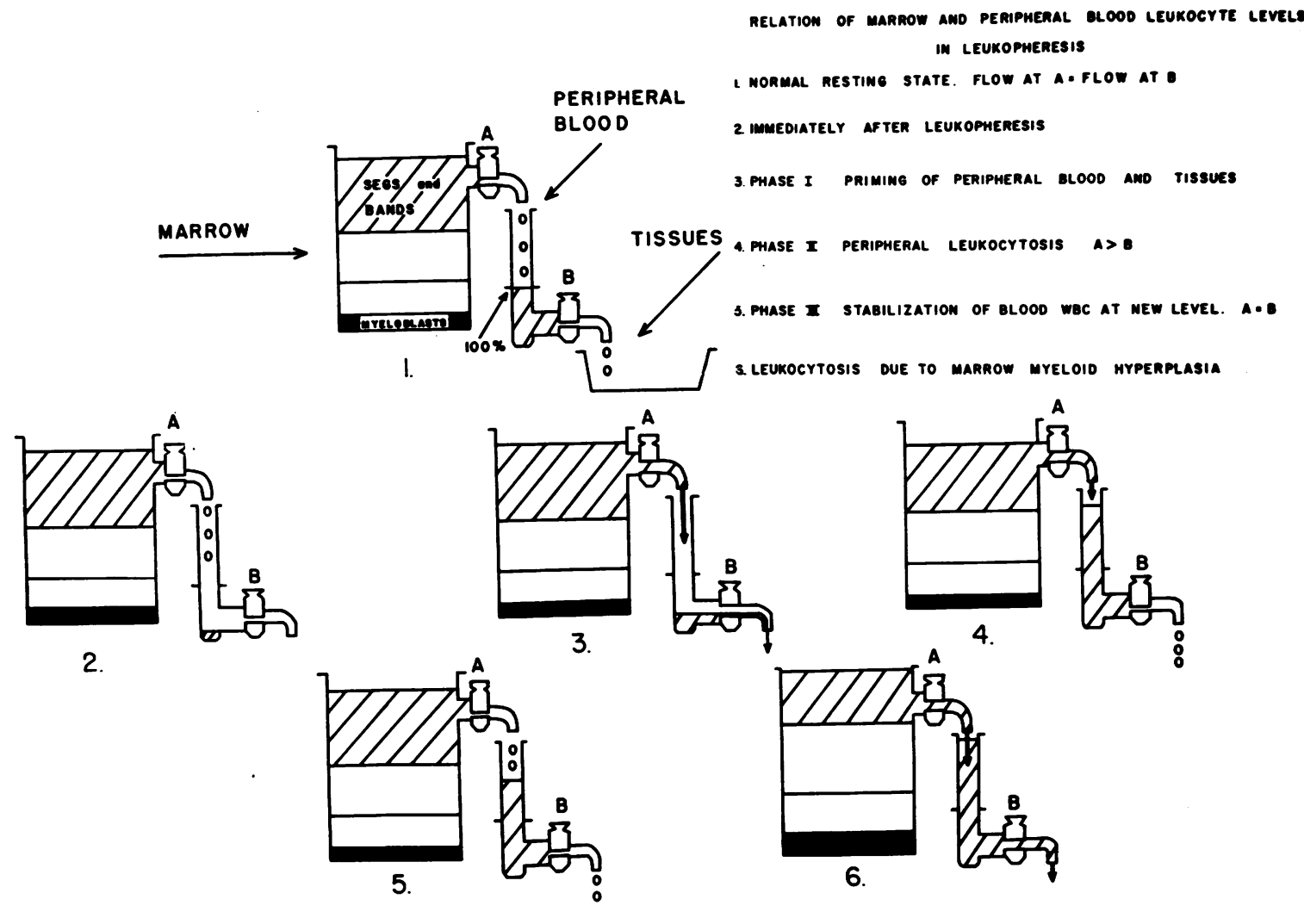

Fig. 14. Diagram

cytes in the periphery. Such utilization may involve actual migration of cells from the blood into the tissues or destruction of leukocytes in the circulation by agglutination, lysis, etc. The leukocyte count at a given moment does not reveal whether the rate of utilization is high, low, or normal, and hence cannot yield information as to the rate of entry of leukocytes from the marrow. However, a persistently elevated neutrophile count must reflect an adequate marrow reserve of leukocytes. Conversely, a low neutrophile count, despite obvious stimulation to neutrophilic leukocytosis, must mean a depleted marrow reserve, as in the leukopenia of overwhelming pyogenic infection. Presumably in this latter situation the continued demand for leukocytes in the periphery outstrips the ability of the growth processes to replace those cells released from the marrow reservoir at a rate sufficient to maintain the volume of the marrow reserve. This phenomenon could result from a great demand for leukocytes imposed on a marrow whose reserves were already depleted by previous disease even if the actual rate of cell synthesis were continuing at a normal or accelerated rate. A small percentage of marrow myeloid cells are myeloblasts, and since these represent the ultimate source of mature leukocytes, depletion of the marrow of its contents of mature cells requires time for replacement. Assuming that the level of peripheral leukocytes in the presence of a stimulus to leukocytosis depends upon the size of the marrow reserve, as suggested by our experiments, the blood leukocyte level will remain low despite intense leukopoiesis if the reserve has been greatly reduced.

Looked upon in this way, the large size of the marrow reserve of mature neutrophilic leukocytes in proportion to those circulating becomes an integral factor in leukocyte physiology. The presence of this reserve, described so clearly by Kindred and Yoffey $(3,4)$, is necessary to prevent the occurrence of marrow depletion by the sudden imposition of a greatly increased rate of peripheral utilization of leukocytes. The formation of new leukocytes is sufficient to balance the demand for leukocytes under resting conditions. However, one can visualize a situation where growth would be inadequate to sustain the need 
for myeloid leukocytes if a sudden demand, many times greater than the resting requirement, were imposed before marrow myeloid hyperplasia had taken place. The marrow reserve affords a huge buffer between supply by growth, which is fixed within certain limits, and demand, which may vary widely. The rate and degree of replenishment of the peripheral blood with leukocytes under conditions of increased need is initially geared to the marrow reserve and not to the rate of growth of myeloid tissue. Thus, heavily irradiated animals respond to leukopheresis with a rapid and marked leukocytosis, until the marrow reserves become depleted. Even after marked depletion of the marrow and peripheral leukopenia, the stimulus associated with leukopheresis leads to release of cells from the marrow, but the rise peripherally is very slow and the maximum is very low. The mechanisms involved in the accelerated release of cells from the marrow reserve induced by leukopheresis remain to be investigated. Whether the primary stimulus is a change in the level or concentration of leukocytes in the peripheral blood or the actual increased removal of cells is not entirely clear. However, the results in irradiated dogs (see Figure 5, for example) suggest that the removal of cells represents a stimulus over and above that of leukopenia itself. Thus, in this animal who was leukopenic at the time leukopheresis was instituted $\left(2,070\right.$ per $\left.\mathrm{mm}^{3}\right)$, the marrow responded by releasing cells more rapidly until a new maximum was reached. The mode of transmission of the stimulus to the marrow resulting in accelerated release of leukocytes from the reserve is presumed to be humoral. However, attempts to detect such a humoral agent have been fruitless thus far.

These experiments have provided some background for further study of leukocyte physiology. It is hoped that the information relating leukopoiesis, marrow reserve and peripheral blood leukocytes will aid those interested in derangements in leukopoiesis and leukemia.

\section{SUMMARY}

1. The concept is presented that the majority of leukocytes which contribute to an acute leukocytosis are released from areas of storage. Increased production of leukocytes by acceleration of leukopoiesis occurs only after considerable delay, which in the case of the dog subjected to repeated leukopheresis is about three to four days.

2. Evidence is presented that the chief source of mature leukocytes taking part in an acute leukocytosis, such as that observed following leukopheresis, is the marrow reserve. This evidence was obtained by taking advantage of the delay before leukocytes whose DNA becomes labeled with $\mathrm{P}^{32}$ during growth move from the marrow into the peripheral blood.

3. Preliminary results of the rate of DNA synthesis in dog neutrophile leukocytes are presented, employing a new method of desoxyribose nucleic acid extraction. These results suggest a shorter life span for this cell in the dog than that reported by others for man.

4. The data are briefly discussed and a concept is formulated which clarifies the relationship of the marrow reserve of leukocytes to those in vascular and extravascular areas.

\section{ACKNOWLEDGMENTS}

Sincere appreciation is expressed for the unlimited help given by Mr. Max Field and Miss Panchita Thomas of the Radioisotope Unit, Research Division, Sawtelle Veterans Administration Hospital under the direction of Dr. Franz Bauer. We are also indebted to Dr. Norman Simmons for providing us with his method of DNA extraction and to Dr. Gian D. Turco for his chromatographic analyses.

\section{REFERENCES}

1. Lawrence, J. S., Physiology and functions of the white blood cells. The Minot Lecture. J.A.M.A., 1955, 157, 1212.

2. Craddock, C. G., Jr., Adams, W. S., Perry, S., Skoog, W. A., and Lawrence, J. S., Studies of leukopoiesis. The technique of leukopheresis and the response of myeloid tissue in normal and irradiated dogs. J. Lab. \& Clin. Med., 1955, 45, 881.

3. Kindred, J. E., A quantitative study of the hemopoietic organs of young adult albino rats. Am. J. Anat., 1942, 71, 207.

4. Yoffey, J. M., The quantitative study of the leukocytes. Ann. New York Acad. Sc., 1955, 59, 928.

5. Osgood, E. E., Number and distribution of human hemic cells. Blood, 1954, 9, 1141.

6. Kline, D. L., and Cliffton, E. E., Life span of leucocytes in man. J. Applied Physiol., 1952, 5, 79.

7. Ottesen, J., On the age of human white cells in peripheral blood. Acta physiol. Scandinav., 1954, 32, 75.

8. Drasher, M. L., A criticism of the indiscriminate use of the Schmidt-Thannhauser method for the fractionation of nucleic acids in biological material. Science, 1953, 118, 181. 